Enfermagem Brasil 2016;15(4):190-7

\title{
ARTIGO ORIGINAL \\ Assistência de enfermagem frente ao paciente com acidente vascular encefálico no setor de emergência
}

Rochelles Spader Prudêncio*, Luciane Bisognin Ceretta, D.Sc. ${ }^{* *}$, Maria Tereza Soratto, M.Sc. ${ }^{*}$

*Enfermeira Pós-Graduada em Assistência de Enfermagem em Urgência e Emergência na Universidade do Extremo Sul de Santa Catarina (UNESC), Criciúma SC, ${ }^{*}$ Enfermeira, UNESC

Recebido em 23 de fevereiro de 2015; aceito em 25 de fevereiro de 2016.

Endereço para correspondência: Maria Tereza Soratto, Rua Dom Joaquim Domingos de Oliveira, 50/301, Ed Jatobá, Centro 88801-230 Criciúma SC, E-mail: guiga@unesc.net, rochelles_sp@hotmail.com, luk@unesc.net

\section{Resumo}

O Acidente Vascular Encefálico (AVE) é uma das maiores causas de atendimentos no setor de emergência hospitalar. Este estudo teve como objetivo identificar a assistência de enfermagem frente ao paciente com AVE no setor de emergência. Pesquisa de abordagem qualitativa, descritiva, exploratória e de campo. O estudo foi desenvolvido em um hospital do Extremo Sul Catarinense. A população estudada foi composta por 5 enfermeiros da emergência do hospital pesquisado. A análise de dados foi realizada a partir da análise de conteúdo. A assistência de enfermagem relacionada à avaliação e monitorização das funções fisiológicas e administração de medicamentos precisa ser rápida, eficaz e qualificada para prevenir complicações tardias e sequelas no paciente. Sugere-se capacitação da equipe de enfermagem, além da organização de um protocolo de acolhimento com classificação de risco e assistência de enfermagem ao paciente com AVE atendido na emergência, de acordo com as diretrizes propostas pelo Ministério da Saúde.

Palavras-chave: assistência de enfermagem, administração dos cuidados ao paciente, acidente vascular encefálico, tratamento de emergência.

\begin{abstract}
Nursing care to patients with stroke in the emergency department

Stroke is one of the major causes of attendances in hospital emergency department. This study aimed to identify the nursing care to patients with stroke in the emergency department. This is a qualitative, descriptive, exploratory and field research. The study was conducted in a hospital in the southern of Santa Catarina. The studied population was composed by 5 nurses working in the researched hospital. Data analysis was carried out using content analysis. Nursing care related to evaluation, follow-up of physiological functions and drug administration should be rapid, efficient and qualified, in order to prevent late complications and sequelae to the patient. We suggest nursing staff qualification, in addition to an organization of a hosting protocol with risk classification and nursing care to the patient with stroke attending emergency department, in accordance with the guidelines proposed by the Health Ministery.
\end{abstract}

Key-words: nursing care, patient care management, stroke, emergency treatment.

\section{Resumen \\ Atención de enfermería a pacientes con accidente cerebrovascular en el departamento de emergencia}

El accidente cerebrovascular (ACV) es una de las mayores causas de atención en los servicio de urgencias hospitalario. Este estudio tiene como objetivo identificar los cuidados a pacientes con ACV en el departamento de emergencia. Se trata de una investigación cualitativa, descriptiva, exploratoria y de campo. El estudio se llevó a cabo en un hospital en sur de Santa Catarina. La población estudiada fueron 5 enfermeros que trabajaban en el hospital del estudio. Los datos de análisis se recolectaron a partir delanálisis de contenido. La atención de enfermería al paciente referente a la evaluación, al seguimiento de funciones fisiológicas y a la administración de medicamentos debe ser rápida, eficaz y de calidad, para prevenir complicaciones tardías y secuelas al paciente. Se sugiere capacitación del personal de 
enfermería, además de organización de un protocolo de clasificación de riesgo y atención de enfermería al paciente con ACV en la sala de emergencia, de conformidad con las directrices propuestas por el Ministerio de la Salud.

Palabras-clave: atención, gestión de atención al paciente, accidente cerebrovascular, tratamiento de emergencia de enfermería.

O Acidente Vascular Encefálico (AVE) é considerado uma doença silenciosa do século que tem maior impacto de mortalidade e morbidade de doenças vasculares, constituindo a primeira causa de incapacitação funcional no mundo e a primeira causa de morte no Brasil [1].

As doenças cerebrovasculares estão no segundo lugar no topo de doenças que mais acometem vítimas com óbitos no mundo, perdendo a posição apenas para as doenças cardiovasculares [2].

No AVE ocorre uma perda súbita do suprimento sanguíneo a uma parte do cérebro. Suas complicações vão além de uma alta taxa de mortalidade, até um grande impacto psicoemocional devido a sequelas neurológicas provocadas no paciente. Desta forma, considera-se de suma importância a capacitação dos enfermeiros e da equipe de enfermagem no processo de atendimento ao paciente com AVE no setor de emergência [3].

O enfermeiro da emergência destaca-se através da escuta da queixa e expectativas dos usuários; identificando as vulnerabilidades e os riscos. Diante disso, percebe-se que a equipe de enfermagem precisa ser amparada por um enfermeiro qualificado, para realizar todos os procedimentos necessários diante da situação presenciada [4].

Estar na emergência significa estar no ambiente de atendimento de urgência e emergência e nos colocarmos em um dos mais difíceis e diversificados momentos de situações opostas de saúde e doença com situações de ambiguidades de sentimentos emoções. $O$ trabalho do enfermeiro passa a envolver todos os aspectos no seu cotidiano, observando cada paciente através de sua convivência, afetividade, integralidade da assistência, proporcionando um clima de confiança, a fim de expressarem muitas vezes suas apreensões e inquietudes, fazendo com que a situação encontrada se torne menos traumática [5].

As doenças cardiovasculares são frequentemente atendidas no setor de emergência, sendo o AVE considerado a primeira causa de incapacitação funcional e morte no Brasil $[1,6]$.

Diante deste panorama surgiram inquietações referentes à assistência de enfermagem ao paciente com AVE, pois o mesmo pode gerar complicações e sequelas no paciente, o que demanda um atendimento eficaz e diferenciado dos enfermeiros. A partir desta realidade resolveu-se conhecer os desafios enfrentados pelos enfermeiros frente a esses pacientes acometidos por AVE no setor de emergência.

O artigo teve como objetivo identificar de que forma ocorre à assistência de enfermagem frente ao paciente com AVE no setor de emergência de um hospital do Extremo Sul Catarinense.

Material e métodos

Pesquisa de abordagem qualitativa, descritiva, exploratória e de campo. O estudo foi desenvolvido em um Hospital do Extremo Sul Catarinense. A população estudada foi composta de 5 enfermeiros da emergência do Hospital pesquisado. A análise de dados foi realizada a partir da análise de conteúdo, através da categorização dos dados [7].

Para preservar o sigilo decorrente da aplicação das entrevistas realizadas com os enfermeiros, de acordo com as diretrizes e normas regulamentadoras da Res. 466/12 [8] que envolvem pesquisa com Seres Humanos, utilizou-se indicador alfanumérico (E1 a E5). A pesquisa foi aprovada pelo Comitê de Ética em Pesquisa da UNESC pelo Projeto no $780.068 / 2014$.

Resultados e discussão

Perfil dos enfermeiros que atuam na emergência hospitalar 
Dos enfermeiros entrevistados quatro (04) são do sexo feminino, e um (01) do sexo masculino, com idade entre 25 e 48 anos. O tempo de trabalho na profissão variou de 1 a 27 anos e no setor de emergência de 1 a 14 anos.

Quanto à especialização, duas (02) enfermeiras realizaram pós-graduação em UTI, urgência e emergência e uma delas apresenta também em Obstetrícia, um (01) enfermeiro apresentou pós-graduação em saúde pública e metodologia da pesquisa, e duas (02) não realizaram especialização até o momento.

Quadro 1 - Perfil dos enfermeiros que atuam na emergência hospitalar.

\begin{tabular}{|l|l|lll|l|}
\hline Profissional & Sexo & Idade & Especialização & $\begin{array}{l}\text { Tempo de } \\
\text { trabalho na } \\
\text { profissão }\end{array}$ & $\begin{array}{l}\text { Tempo de } \\
\text { trabalho no } \\
\text { setor }\end{array}$ \\
\hline E1 & Feminino & 27 & Não possui 5 anos & 1 ano \\
\hline E2 & Feminino & 28 & $\begin{array}{l}\text { UTI. Urgência e } 6 \text { anos } \\
\text { Emergência. } \\
\text { Obstetrícia. } \\
\text { UTI. Urgência e } 4 \text { anos } \\
\text { Emergência. }\end{array}$ & 6 anos \\
\hline E4 & Feminino & 25 & anos & 14 anos \\
\hline E5 & Feminino & 30 & 48 & $\begin{array}{l}\text { Saúde pública. } \\
\text { Metodologia do } \\
\text { Ensino. }\end{array}$ & Não possui. 9 anos \\
\hline
\end{tabular}

Fonte - Dados da pesquisa, 2014.

\section{Capacitação para atender pacientes com AVE no setor de emergência}

O estudo apresentou ponto negativo referente à capacitação dos enfermeiros, pois todos os entrevistados responderam que não receberam capacitação para atender pacientes que apresentam AVE no setor de emergência, sobretudo afirmaram que o conhecimento que possuem se dá somente mediante suas práticas diárias e seus embasamentos teóricos adquiridos na graduação.

Os profissionais de enfermagem, sobretudo os enfermeiros, que atuam nos setores de emergência são essenciais no processo de cuidar. É necessário que tenham competência, destreza, cuidado holístico, criatividade e sensibilidade [9].

Considera-se imprescindível a qualificação e atualização, específica e continuada, do Enfermeiro para atuar no processo de classificação de risco e priorização da assistência à saúde [10].

Portanto é de suma importância que o enfermeiro tenha capacitação e qualificação específica para atender pacientes que apresentam AVE, para que os mesmos possam desenvolver com habilidade todas as situações encontradas, proporcionando ao paciente uma possível diminuição das sequelas e o risco eminente de morte.

\section{Os tipos de AVE mais atendidos no setor de emergência}

Segundo a Organização Mundial de Saúde (OMS), "o AVE é uma síndrome clínica que consiste no desenvolvimento rápido de distúrbios clínicos focais da função cerebral que duram mais de 24 horas ou conduzem à morte sem outra causa aparente que não uma de origem vascular" [6].

Dentre as principais manifestações clínicas, os sinais neurológicos variam conforme a localização e o tamanho da lesão cerebral. Na maioria dos casos os pacientes apresentam dormência ou fraqueza da face, braço ou perna, principalmente em um lado do corpo, apresentam também confusão ou alteração no estado mental, problema ao proferir ou compreender a fala, distúrbios visuais, dificuldade em deambular, perda do equilíbrio e cefaleia intensa [1]. 
Os acidentes vasculares encefálicos podem ser divididos em duas categorias, como isquêmicos, quando ocorre uma oclusão vascular e uma hipoperfusão significativa, e os hemorrágicos, quando ocorre extravasamento de sangue para o cérebro ou para o espaço subaracnóide [3].

De acordo com a vivência dos entrevistados, todos relataram que o AVE isquêmico ocorre com mais frequência no setor de emergência, confirmado com a literatura, cujas pesquisas mostram que aproximadamente $80 \%$ dos AVCs são causados por um baixo fluxo sanguíneo cerebral (isquemia) e outros $20 \%$ por hemorragias intraparenquimatosas ou subaracnóideas $[6,11]$.

No AVE isquêmico ocorre interrupção do fluxo sanguíneo, causado por estenose carotídea, pelo estreitamento da carótida ou pela presença de coágulos deslocados para o cérebro. Já, por sua vez, o AVE hemorrágico ocorre quando há o extravasamento do sangue e subdivide-se em Hemorrágico Intracerebral (sangramento de uma das artérias do cérebro para o tecido cerebral) ou Hemorrágico Subaracnóideo (no qual a hemorragia se desloca entre as meninges Pia-máter e a Aracnóide)[3].

Essa classificação se torna de extrema importância, pois, segundo OMS, o risco de morte depende do tipo de AVE. Em ambos, o atendimento adequado pode depender da extensão e do local das lesões, determinante na reversibilidade do AVE [3].

\section{Protocolo de acolhimento com classificação de risco}

No Brasil os serviços hospitalares, especialmente os setores de emergência têm se caracterizado cada vez mais por longas filas de espera. De acordo com essa realidade, 0 Ministério da Saúde tem reafirmado a Política Nacional de Humanização da Atenção e Gestão do SUS (Humaniza SUS), no qual faz parte o acolhimento com classificação de risco, com o objetivo de melhorar a qualidade e agilidade de atendimento do Sistema Único de Saúde [12].

Os enfermeiros entrevistados citaram que não existe protocolo de acolhimento com classificação de risco na urgência e emergência; no entanto, segundo os enfermeiros E1 e E2, existe projeto para implantação do protocolo.

E1 A E2: "Não existe, mas já possui projeto para implantação".

E3 a E5: "Não".

Um desafio citado pelo estudo é a implantação do acolhimento com classificação de risco cujo projeto já foi realizado, aguardando aprovação. Devido ao aumento da demanda dos atendimentos, muitos pacientes não sabem como se dirigir até a sala de emergência ou, muitas vezes, são encaminhados sem ter necessidade de atendimento imediato.

\section{O acolhimento com classificação de risco na emergência}

Ao questionar os enfermeiros de que forma é realizado o acolhimento com classificação de risco E1, E2 e E5 relataram que são as recepcionistas que encaminham os pacientes determinando o grau de complexidade de cada indivíduo, e os demais entrevistados E3 e E4 referiram que estes pacientes quando dão entrada na emergência vêm acompanhados dos bombeiros ou de serviço de atendimento móvel de urgência (SAMU).

E1: "A recepcionista encaminha direto para a sala de emergência de acordo com a apresentação dos sintomas emergenciais".

E2: "Existem duas portas na recepção, uma entrada para a recepcionista, outra para a emergência. Alguns pacientes vão até a recepcionista referindo o problema, outros se dirigem a sala de emergência".

E5: "A recepcionista encaminha até a sala de emergência".

E3: "Geralmente os pacientes com AVE vêm acompanhados dos bombeiros ou SAMU, daí encaminhados diretamente à sala de emergência".

E4: "Bombeiros e SAMU transportam até a sala de emergência".

A classificação de risco e correspondente priorização do atendimento em Serviços de Urgência caracterizam-se como um processo complexo, que demanda competência técnica e científica em sua execução. O processo de acolhimento e classificação de risco é considerado como parte do sistema de humanização da assistência, objeto de padronização do Ministério da Saúde [10]. 
A metodologia internacionalmente reconhecida para classificação de risco (Protocolo de Manchester) prevê que o usuário seja acolhido por uma equipe que definirá o seu nível de gravidade e o encaminhará ao atendimento específico de que necessita [10].

$\mathrm{O}$ acolhimento no setor de emergência deve ser realizado pelo enfermeiro, através da humanização, garantindo acesso a todas as pessoas. Diz respeito ainda à escuta qualificada de problemas de saúde do usuário, visando fornecer sempre uma resposta positiva. Já a classificação de risco implica a agilidade do atendimento mediante a aplicação de um protocolo que determina o grau da necessidade do usuário, conforme a complexidade e não a ordem de chegada [12].

Segundo a Resolução COFEN no 423/2012 [10], no âmbito da equipe de Enfermagem, a classificação de risco e priorização da assistência em Serviços de Urgência é privativa do Enfermeiro, observada as disposições legais da profissão. Para executar a classificação de risco e priorização da assistência, o enfermeiro deverá estar dotado dos conhecimentos, competências e habilidades que garantam rigor técnico-científico ao procedimento [10].

\section{Assistência de enfermagem frente ao paciente com AVE}

Foram citados por todos os profissionais que a assistência de enfermagem ao paciente que dá entrada na emergência está relacionada inicialmente aos aspectos biológicos dos pacientes, como avaliação e monitorização das funções fisiológicas e administração de medicamentos.

\footnotetext{
E1: "Primeiramente verifica-se os sinais vitais, punciona-se acesso venoso, verifica-se se o paciente já apresenta alguma patologia, monitora-se, e após atendimento médico agenda-se a tomografia".

E2: "Estabiliza-se o paciente com acesso venoso, monitorização cardíaca, após atendimento médico seguir cuidados específicos para a patologia".

E3: "Acesso venoso periférico, administrar drogas CPM, fornecer materiais adequados conforme solicitação".

E4: "Verifica-se sinais vitais, punciona-se acesso venoso de grosso calibre, realiza-se glicemia capilar, e deve-se ficar atento a todos os sinais e sintomas apresentados".

E5: "Verifica-se sinais vitais, acesso venoso periférico, sondagem (SN), comunicar o laboratório se forem solicitados exames, acomodar paciente no leito".
}

A avaliação inicial e assistência de enfermagem ao paciente que apresenta AVE no setor emergência deverão ser realizadas pelo enfermeiro e deve-se enfocar na avaliação das vias aéreas, circulação, respiração, sinais vitais e exame neurológico $[1,13]$.

$\mathrm{Na}$ maioria dos casos, os indivíduos com AVE procuram a emergência para a realização de investigações acerca da origem, gravidade e grau de comprometimento das funções corporais, para posteriormente, realizarem o tratamento e prevenção das possíveis sequelas [14]. Portanto, quanto mais cedo se inicia o processo de reabilitação, maiores serão as chances de o indivíduo ter uma recuperação rápida e completa, favorecendo seu prognóstico.

A reabilitação é uma das inúmeras funções da enfermagem, que busca no indivíduo a independência para a realização do autocuidado. A habilidade para realizá-lo é frequentemente a chave para a independência, para o retorno ao lar e para sua vida comunitária [14].

Considera-se importante informar aos pacientes acometidos por AVE que poderá haver recuperação total ou parcial, porém essa evolução dependerá de alguns fatores, tais como, a extensão, o local da lesão cerebral, a idade, e o tempo que levou para ser prestado o atendimento de emergência.

Além dos cuidados emergenciais e aqueles durante o período de internação, alguns estudos revelam que o adequado planejamento da alta hospitalar pode favorecer a melhoria da qualidade, da continuidade do tratamento, devido à maioria dos pacientes necessitarem de um cuidado domiciliar posteriormente [14].

A enfermagem tem se destacado como uma profissão de importante proximidade com o paciente e, por isso, é responsável por um olhar holístico no processo de cuidar. Sob esta percepção é de suma importância que o enfermeiro ofereça todo o suporte e assistência de enfermagem, desde a promoção de saúde até sua reabilitação [15].

Relacionado à assistência de enfermagem diante de algumas alterações apresentadas devido a sequelas do AVE, principalmente o controle muscular e a sensibilidade corpórea, orienta-se o paciente que deve manter um posicionamento anatômico correto, fazer uso de almofadas e travesseiros, pois o posicionamento inadequado pode gerar desconforto e dor. Além disso, orienta-se também que faça alongamentos, com a finalidade de manter a 
amplitude de movimentação dos membros, porém estar atento quanto à prevenção de possíveis quedas, se necessário inicialmente fazer uso de andador e bengala [14].

Portanto o enfermeiro deve ser capaz de reconhecer os sintomas neurológicos que sugerem AVE e rapidamente analisar o tempo inicial dos sintomas, até que se tenha um atendimento e um diagnóstico médico.

Diante deste panorama, o enfermeiro deve estar apto e atualizado para atender os pacientes frente a esta situação [1].

\section{Facilidades e dificuldades encontradas no atendimento ao paciente com AVE}

Os enfermeiros citaram como facilidades a unidade de estabilização associado aos recursos materiais e medicações. $\mathrm{O}$ enfermeiro $\mathrm{E} 2$ ainda citou a equipe de enfermagem como facilidade para o atendimento ao paciente com AVE.

\footnotetext{
E1: "Facilidades: Possuem quase todos os recursos, como aparelhos, medicações".

E2: "Facilidades: Possui uma unidade de estabilização ampla, com ventiladores mecânicos, bombas de infusão, monitor cardíaco, equipe de enfermagem (técnico e enfermeiro)".

E3: "Facilidades: Boas referências para transferência, disponibilização de todas as medicações necessárias para o atendimento".

E4: "Facilidades: Oferta de aparelhos, como oxigênio, oxímetro, e medicações".

E5: "Facilidades: Unidade de estabilização, como monitores, respiradores, medicações".
}

A maioria dos enfermeiros considera como dificuldades a transferência do paciente para o hospital de referência, a exceção do enfermeiro 3 que destaca a inexperiência médica:

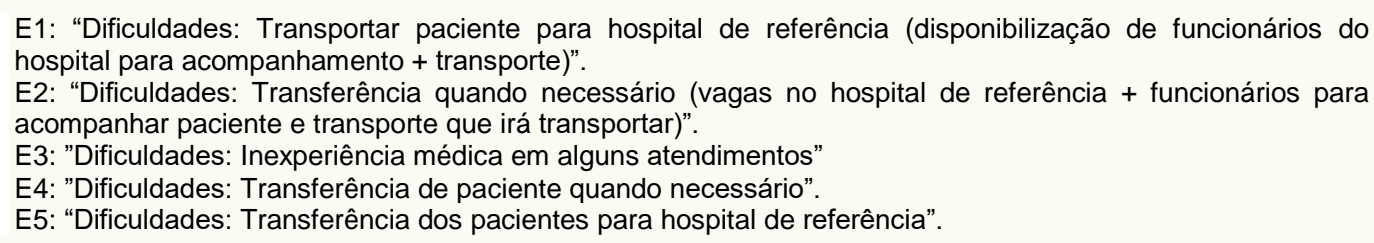

O tratamento adequado e inicial para o paciente com AVE seria salvar o máximo possível da área isquêmica atingida, se possível deverá ser iniciado dentro da janela terapêutica até três horas do início dos sinais e sintomas apresentados. Já o objetivo do tratamento do AVE hemorrágico é permitir que o cérebro recupere sua lesão inicial para prevenir um novo sangramento [6].

O tratamento recomendado consiste na utilização das novas tecnologias na fase aguda, seguindo rigorosos protocolos de segurança, que devem ser seguidas por equipe multidisciplinar coordenada e treinada. Essas unidades devem dispor de equipamentos como a tomografia computadorizada, ressonância magnética, ecodoppler, suporte de transporte, exames laboratoriais, além de ações educativas contínuas para profissionais de saúde e população [6].

\section{Orientações prestadas aos pacientes e familiares durante o atendimento e após alta hospitalar}

De acordo com o grau de comprometimento da lesão, o paciente poderá desenvolver diversas complicações, como alterações comportamentais cognitivas, disfasia, constipação intestinal, epilepsia vascular, depressão e outras implicações decorrentes da imobilidade e acometimento muscular [6].

Diante das orientações prestadas aos pacientes e familiares, durante o atendimento e após a alta hospitalar, percebeu-se que os profissionais em estudo realizaram suas orientações sobre a conduta adequada, expondo de que maneira o atendimento deve ser seguido conforme o estado em que o paciente se encontra.

E1: "Sobre os cuidados de saúde e de reabilitação que o paciente irá necessitar de acordo com a situação, como a importância da realização de fisioterapia. Orientar também sobre o apoio da secretaria de saúde do município de referência no cuidado domiciliar".

E2: "Sobre os cuidados que deverá seguir como higiene, locomoção, mudança de decúbito e fisioterapia".

E3: "Orientar para manter repouso no leito, procurar serviços de fisioterapia, nutrição e também orientar a procurar se necessário a secretaria de saúde para acompanhamento do caso ". 
E4: "Orientar sobre a higiene e conforto / higiene oral, cuidados com sonda nasogástrica ou nasoenteral (SN), cuidados com área de pressão, realizar mudança de decúbito ".

E5: "Orientações conforme o estado em que o paciente se encontra como deglutição, higiene, locomoção, fisioterapia, mudança de decúbito".

Vale ressaltar que, na maioria das vezes, para que o paciente possa ter uma melhor recuperação e qualidade de vida, é fundamental que ele seja analisado e acompanhado por uma equipe multidisciplinar de profissionais da saúde, além dos cuidados de enfermagem, com médico neurologista, fisioterapeuta, nutricionista, terapeuta ocupacional e psicólogo. Por isso, reforçou-se a necessidade de orientação quanto aos serviços de atenção básica encontrados na secretaria de saúde do município para acompanhamento domiciliar.

\section{Sugestão sobre o tema - Assistência de enfermagem frente ao paciente com AVE}

As sugestões ressaltadas pela maioria dos entrevistados foram relacionadas à necessidade de capacitações para os profissionais atuantes na emergência, já que o estudo referiu déficit de uma educação continuada.

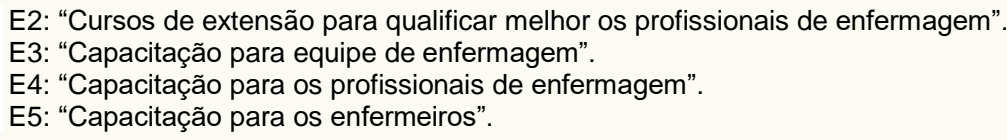

Somente um enfermeiro (E1), citou como importância, uma política de prevenção aos acidentes vasculares encefálicos: E1: "Sobre uma política de prevenção relacionada à patologia".

A Linha do Cuidado do AVC, instituída pela Portaria MS/GM no 665, de 12 de abril de 2012, e parte integrante da Rede de Atenção às Urgências e Emergências, propõe uma redefinição de estratégias que deem conta das necessidades específicas do cuidado ao AVC [2].

Os serviços de saúde autorizados a prestar assistência aos pacientes acometidos por AVC no âmbito do SUS deverão observar a Linha de Cuidados em AVC e o Protocolo Clínico e Diretrizes Terapêuticas do Acidente Vascular Cerebral Isquêmico Agudo, instituído por meio da Portaria no 664/GM/MS, de 12 de abril de 2012 [16]. Ainda recomenda-se ao serviço de emergência basear-se no protocolo descrito no Manual de Rotinas para atenção ao AVC [17].

\section{Conclusão}

O AVE é uma das maiores causas de atendimentos no setor de emergência hospitalar, destacando-se o AVE isquêmico como o de maior frequência.

A assistência de enfermagem rápida, eficaz e qualificada previne complicações tardias e sequelas no paciente. A orientação ao paciente e seus familiares é essencial para o processo de recuperação e diminuição da ansiedade dos mesmos, objetivando diminuição das complicações e sequelas.

Os enfermeiros citaram como facilidades a unidade de estabilização associada aos recursos materiais, medicações e a equipe de enfermagem. Já como dificuldades ressaltaram a transferência do paciente para o hospital de referência e a inexperiência médica em alguns atendimentos.

As sugestões ressaltadas pela maioria dos entrevistados foram relacionadas à necessidade de capacitações para os profissionais atuantes na emergência e a necessidade de uma política de prevenção aos acidentes vasculares encefálicos.

Os enfermeiros não receberam capacitação para atender pacientes que apresentam AVE no setor de emergência, desta forma, sugere-se capacitação da equipe de enfermagem sobre as temáticas:

- Acidente Vascular Encefálico (AVE): conceito, fatores causais, tipos de AVE, manifestações clínicas, tratamento;

- Acolhimento e Protocolo de Classificação de risco no atendimento ao AVE;

- Assistência de enfermagem no setor de emergência, baseando-se na Portaria no 664/GM/MS, de 12 de abril de 2012, que aprova o Protocolo Clínico DiretrizesTerapêuticas - Trombólise no 
Acidente Vascular Cerebral Isquêmico Agudo; Portaria ํo. 665, de 12 de abril de 2012 sobre Linha de Cuidados em AVC e no Manual de Rotinas para atenção ao AVC.

A partir da capacitação da equipe de enfermagem, o enfermeiro deve realizar 0 acolhimento com classificação de risco aos pacientes com AVE na emergência segundo diretrizes estabelecidas pelo Conselho Federal de Enfermagem.

Sugere-se um aprofundamento da pesquisa com a organização e de um protocolo de acolhimento com classificação de risco e assistência de enfermagem ao paciente com AVE atendido na emergência, de acordo com as diretrizes fundamentadas pelo Ministério da Saúde.

Faz-se necessário que o enfermeiro juntamente com a equipe de enfermagem devem orientar o paciente e seus familiares e cuidadores acerca de informações fundamentais que culminam na realização de uma assistência integral, promovendo uma melhor qualidade de vida aos pacientes que tiveram comprometimento neurológico.

Referências

1. Tavares SMS, Oliveira IRS. O cuidado de enfermagem ao paciente com acidente vascular cerebral em ambiente intra-hospitalar, 2013. [citado 2014 Ago 5]. Disponível em URL: < http://www.redentor.inf.br/arquivos/pos/publicacoes

2. Brasil. Ministério da Saúde. Secretaria de Atenção à Saúde. Departamento de Atenção Especializada. Manual de rotinas para atenção ao AVC. Brasília: Ministério da Saúde; 2013. 50 p.

3. Brunner LS, Suddarth DS. Tratado de enfermagem Médico- Cirúrgica. Rio de Janeiro: Guanabara Koogan; 2009.

4. Panizzon C, Luz AMH, Fensterseifer LM. Estresse da equipe de enfermagem de emergência clínica. Rev Gaúcha Enferm 2008;29(3):391-9.

5. Andrade LM, Martins EC, Caetano JA, Soares E, Beserra EP. Atendimento humanizado nos serviços de emergência hospitalar na percepção do acompanhante. Rev Eletrônica Enferm 2009;1(1):151-7.

6. Leite SMA. Disseminação de informações em ações especificas para AVC [Dissertação]. Rio de Janeiro: Fiocruz; 2009. $83 \mathrm{f}$.

7. Minayo MCS. Pesquisa social: teoria, método e criatividade. 28 ed. Petrópolis: Vozes; 2009. $108 \mathrm{p}$.

8. Brasil. Conselho Nacional de Saúde. Resolução no 466, de 12 de dezembro de 2012. [citado 2014 Jul 13]. Disponível em URL: http://conselho.saude.gov.br

9. Avelar VLLM, Paiva KCM. Configuração identitária de enfermeiros de um serviço de atendimento móvel de urgência. Rev Bras Enferm 2010;63(6).

10. Cofen. Resolução COFEN no 423/2012. [Citado 2015 fev 9]. Disponível em URL: http://www.cofen.gov.br/resoluo-cofen-n-4232012_8956.html

11. Leal MGS. Derrame - acidente vascular cerebral - informações para a família e cuidadores. São Paulo: Novartis Biociências; 2009.

12. Vituri DW. Acolhimento com classificação de risco em hospitais de ensino: avaliação da estrutura, processo e resultado. Rev Latinoam Enferm 2013;21(5):1-9.

13. Cavalcante TF, Moreira RP, Guedes NG, Araujo TL, Lopes MVO, Damasceno MMC et al. Intervenções de enfermagem aos pacientes com acidente vascular encefálico: uma revisão integrativa de literatura. Rev Esc Enferm USP 2011;45(6):1495-500.

14. Lessmann JC, Conto FD, Ramos G, Borenstein MS, Meirelles BHS. Atuação da enfermagem no autocuidado e reabilitação de pacientes que sofreram Acidente Vascular Encefálico. Rev Bras Enferm 2011;64(1):198-202 .

15. Nascimento LC, Santos TFM, Oliveira FCS, Pan R, Santos MF, Rocha SMM. Espiritualidade e religiosidade na perspectiva de enfermeiros. Texto Contexto Enferm 2013;22(1):52-60.

16. Brasil. Ministério da Saúde. Portaria no 664, de 12 de abril de 2012. [citado 2015 fev 9]. Disponível em URL: http://bvsms.saude.gov.br

17. Brasil. Ministério da Saúde. Portaria no. 665, de 12 de abril de 2012. Aprova a linha de cuidados em AVC. [citado 2015 Fev 9]. Disponível em URL: http://bvsms.saude.gov.br/bvs. 
